\title{
Dyspnea And Quality of Life in Patients Referred For Lung TRANSPLANTATION
}

\author{
W. Lutogniewska ${ }^{1}$, D. Jastrzebski1 ${ }^{1}$, J. Wyrwol ${ }^{1}$, B. Ksiazek ${ }^{1}$, M. Ochman², K. Kowalski1 ${ }^{1}$, A. Margas ${ }^{1}$, \\ P. Kubicki ${ }^{3}$, J. Kozielski ${ }^{1}$ \\ ${ }^{1}$ Department of Lung Diseases and Tuberculosis, Silesian Medical University, Zabrze, Poland; \\ ${ }^{2}$ Department of Transplantology, Silesian Center for Heart Diseases, Zabrze, Poland; \\ ${ }^{3}$ Academy of Physical Education, Physical Education Faculty, Katowice, Poland
}

\begin{abstract}
Background: In the years 2007-2010 in the Department of Lung Diseases and Tuberculosis, Medical University of Silesia, 86 patients fulfilling ISHLT criteria qualified for lung transplantation.

Objectives: The aim of the study was to assess the correlation between dyspnea and quality of life, and how it is related to clinical data in the examined group.

Material and methods: MRC, OCD, BDI and Borg scale were used for dyspnea evaluation, whereas quality of life was evaluated with SF-36 and SGRQ. A reference group consisted of 18 females and 68 males of the mean age $52 \pm 10$ years and BMI $24 \pm 6$. Thirty patients were diagnosed with IPF, 22 with COPD, and 34 with IIP.

Results: In the reference group, there was a significant correlation between dyspnea and quality of life: between MRC and Pf (SF-36 domain) $\mathrm{r}=-0.53$; OCD and activity (SGRQ) $\mathrm{r}=0.56$; OCD and Pf $\mathrm{r}=$ -0.55 ; BDI and impact (SGRQ) $r=0.51$; Borg scale and impact $r=0.47$. In patients after lung transplantation, correlation between MRC and SF was $r=-0.92$; OCD and Pf, Bp, MH, PCS $\mathrm{r}=-0.97$; OCD and RE $r=-0.89$; BDI and Pf $r=-0.89$; BDI and activity $r=0.9$; BDI and PCS $r=-0.84$. Depending on the diagnosis, the strongest correlation in IIP patients was found between OCD and activity $(r=0.62)$, in COPD patients - between BDI and impact $(\mathrm{r}=0.79)$, and in IPF patients $r=-0.62$ for OCD and Pf. Summing up the results, we can state that there is a significant correlation between dyspnea and quality of life. This correlation seems the strongest in patients after lung transplantation.

Conclusions: The correlation found between the level of dyspnea and quality of life domains in lung transplant patients suggests that it would be worthwhile to add questions regarding dyspnea to assess the severity of the disease, clinical symptoms, and functional impairment during referring the patients for lung transplantation.
\end{abstract}

Key words: lung transplantation, dyspnea, quality of life

\section{INTRODUCTION}

The University of Silesia is the only center in Poland, where lung transplantations (LT) are performed. Patients are referred for lung transplantation in the Department of Lung Diseases and Tuberculosis. The selection of patients for lung transplants consists of the estimation of disease progress not only in the respiratory system, but also the condition of other life important organs. The main goal of LT, rather than to increase of life expectancy, is to improve the quality of life (QL). Each patient during referring for LT has an accurate estimation of QL performed. Tests specific for the end-stage, advanced lung diseases have not been invented yet. Among the available tests, the SF-36 questionnaire assesses both physical and mental conditions. Saint Georges Respiratory Questionnaire (SGRQ), specified for chronic lung diseases, evaluates the quality of life in three domains: symptoms, activity, and impact on life. The aim of the present study was to determine the correlation between quality of life and dyspnea in patients awaiting lung transplantation, and to assess whether this correlation could related to the clinical data in the group examined.

\section{MATERIAl AND Methods}

The study was performed in accordance with the Declaration of Helsinki for Human Research and study protocol was approved by the Ethics Committee of the Silesian Medical University in Zabrze, Poland.

In years 2007-2010, eighty six patients fulfilling the ISHLT criteria [1], which qualify for lung transplantation, were referred for the study. The study group consisted of 18 females and 68 males of the mean age 52 \pm 10 years and BMI $24 \pm 6 \mathrm{~kg} / \mathrm{m}^{2}$. Thirty patients were diagnosed with idiopathic pulmonary fibrosis (IPF), 22 with chronic obstructive pulmonary disease (COPD), and 34 with other than IPF forms of idiopathic interstitial pneumonia (IIP). We evaluated the quality of life with two questionnaires: SGRQ and SF-36. Methodological details and description of QL domains have been described in details previously [2].

Dyspnea was evaluated with: Medical Research Council (MRC), Oxygen-Cost Diagram (OCD), Base- 
line Dyspnea Index (BDI), and Borg scale. MRC grades the degree of breathlessness related to activities; OCD - assesses the dyspnea severity by identifying the limits of physical activity due to dyspnea; BDI consists of three elements: FI - functional impairment, MT - magnitude of task, and ME - magnitude of effort. The Visual Borg Scale assesses patient's dyspnea from 0 (rest) to 10 , where 10 is the maximum ever experienced dyspnea.

Results are expressed as means \pm SD. Spearman's rank correlation coefficient was used to measure of statistical dependence. The correlations between the quality of life domains, the results of dyspnea tests and anthropometric data, such as body mass index and age, were determined. Statistical significance was defined as $\mathrm{P}<0.05$.

\section{RESULTS}

\section{Dyspnea Evaluation}

The data on the evaluation of dyspnea, stratified according to the character of a disease process are displayed in Table 1. According to the MRC test, the mean score for dyspnea in the whole study group of patients was 3.6 \pm 0.7 . Dyspnea perception was the strongest in IIP, $3.8 \pm 0.5$, and weakest in IPF patients (3.3 \pm 0.7$)$. Dyspnea in the OCD test was $4.0 \pm 0.9$ in the whole group; it was $4.0 \pm 0.8$ in IIP and $3.9 \pm 1.2$ in COPD patients. According to the BDI test, dyspnea score amounted to $12.6 \pm 2.7$ in the whole group, with rather small differences according to disease stratification. In the Borg visual scale, dyspnea score amounted to $5.9 \pm 2.3$ in the whole; being the highest in IIP, 6.6 \pm 1.8 and lowest in COPD patients, $5.5 \pm 2.5$.

Table 1. Dyspnea evaluation.

\begin{tabular}{lrrrr}
\hline & Total score & IPF & COPD & \multicolumn{1}{c}{ IIP } \\
\hline MRC & $3.6 \pm 0.7$ & $3.3 \pm 0.7$ & $3.5 \pm 0.9$ & $3.8 \pm 0.5$ \\
OCD & $4.0 \pm 0.9$ & $3.9 \pm 0.9$ & $3.9 \pm 1.2$ & $4.0 \pm 0.8$ \\
BDI & $12.6 \pm 2.7$ & $12.8 \pm 1.8$ & $12.9 \pm 1.9$ & $12.4 \pm 3.6$ \\
Borg & $5.9 \pm 2.3$ & $5.6 \pm 2.6$ & $5.5 \pm 2.5$ & $6.6 \pm 1.8$ \\
\hline
\end{tabular}

\section{QuAlity OF LiFE EVALUATION}

In SGRQ, the most impaired domain of quality of life was activity $(74.6 \pm 18.4)$, whereas the least affected were symptoms $(61.0 \pm 20.2)$. The global score indicates the poorest quality of life in IIP patients $(70.1 \pm 12.5)$, whereas the best score was achieved in the group of patients diagnosed with IPF $(64.0 \pm 19.0)$ (Table 2). Quality of life evaluated with the SF-36 questionnaire turned out to be the most impaired in RP domain (16.2 $\pm 22.6)$ and least in BP domain (48.8 \pm 22.1$)$. Based on the cumulative scores, the smallest PCS impairment was discovered in IIP $(26.0 \pm 6.3)$ and biggest $(25.1 \pm 8.3)$ in COPD patients. The mental cumulative health score was the highest in IPF (42.4 \pm 14.2$)$, and lowest in COPD patients $(37.8 \pm 15.3)$ (Table 3$)$.

\section{DysPNEA AND QUALITY OF LIFE}

In the study group, there was a significant correlation between dyspnea and quality of life: between MRC and Pf (SF-36 domain) $\mathrm{r}=-0.53$; OCD and activity (SGRQ) $\mathrm{r}=0.56$; OCD and $\mathrm{Pf} \mathrm{r}=-0.55$; BDI and impact (SGRQ) $r=0.51$; Borg scale and impact $r=0.47$ (Table 4). Depending on the diagnosis, the strongest correlation in IIP patients was found between OCD and activity $(\mathrm{r}=0.62)$, in COPD patients between BDI and impact $(\mathrm{r}=0.79)$, and in IPF patients between OCD and Pf $\mathrm{r}=-0.62$ for (Table 5).

There were no correlations between BMI and dyspnea or BMI and any of the quality of life domains. Nor were there any significant correlations between age and dyspnea (MRC, OCD, BDI, or Borg). However, there were significant correlations between age and

Table 2. Quality of life evaluation - SGRQ.

\begin{tabular}{lcccc}
\hline & Global score & Symptoms & Activity & Impact \\
\hline Total & $67.7 \pm 16.7$ & $61.0 \pm 20.2$ & $74.6 \pm 18.4$ & $70.2 \pm 19.7$ \\
IPF & $64.0 \pm 19.0$ & $58.7 \pm 20.3$ & $70.4 \pm 20.1$ & $65.4 \pm 24.8$ \\
COPD & $68.8 \pm 18.9$ & $62.2 \pm 21.2$ & $74.8 \pm 21.7$ & $71.8 \pm 20.6$ \\
IIP & $70.2 \pm 12.5$ & $62.1 \pm 19.7$ & $78.3 \pm 13.6$ & $73.3 \pm 12.4$
\end{tabular}

Table 3. Quality of life evaluation - SF-36.

\begin{tabular}{lccccc}
\hline & PF & RP & BP & GH & VIT \\
\hline Total & $19.3 \pm 15.9$ & $16.2 \pm 22.6$ & $48.8 \pm 27.1$ & $25.9 \pm 11.9$ & $37.5 \pm 17.0$ \\
IPF & $19.1 \pm 17.0$ & $20.7 \pm 23.2$ & $47.3 \pm 25.9$ & $28.5 \pm 10.7$ & $38.7 \pm 19.8$ \\
COPD & $24.3 \pm 18.8$ & $8.0 \pm 14.0$ & $54.0 \pm 27.2$ & $27.7 \pm 13.3$ & $35.4 \pm 14.4$ \\
IIP & $16.1 \pm 12.1$ & $16.9 \pm 25.1$ & $46.7 \pm 28.5$ & $22.5 \pm 11.4$ & $37.7 \pm 16.2$ \\
& & & & & PCS \\
\hline Total & $33.0 \pm 24.6$ & $35.6 \pm 36.8$ & $47.6 \pm 21.4$ & $25.8 \pm 7.3$ & $39.6 \pm 13.6$ \\
IPF & $33.4 \pm 26.0$ & $40.3 \pm 36.9$ & $49.2 \pm 22.8$ & $25.9 \pm 7.8$ & $42.4 \pm 14.2$ \\
COPD & $37.9 \pm 29.0$ & $32.4 \pm 38.8$ & $51.7 \pm 18.8$ & $25.1 \pm 8.3$ & $37.8 \pm 15.3$ \\
IIP & $29.4 \pm 20.1$ & $33.2 \pm 36.2$ & $43.6 \pm 21.7$ & $26.0 \pm 6.3$ & $38.2 \pm 12.1$ \\
\hline
\end{tabular}


Table 4. Correlations between dyspnea and quality of life.

\begin{tabular}{llrl}
\hline Dyspnea & Quality of life & \multicolumn{1}{c}{$\mathrm{r}$} & $\mathrm{P}$ \\
\hline MRC & PF (SF-36) & -0.53 & $<0.05$ \\
OCD & PF (SF-36) & -0.55 & $<0.05$ \\
OCD & Activity (SGRQ) & 0.56 & $<0.05$ \\
BDI & Impact (SGRQ) & 0.51 & $<0.05$ \\
BORG & Impact (SGRQ) & 0.47 & $<0.05$ \\
\hline
\end{tabular}

Table 5. Correlations between dyspnea and quality of life depending on the diagnosis.

\begin{tabular}{lllrr}
\hline Diagnosis & Dyspnea & Quality of life & $\mathrm{r}$ & $\mathrm{P}$ \\
\hline ILD & OCD & Activity (SGRQ) & 0.62 & $<0.05$ \\
COPD & BDI & Impact (SGRQ) & 0.79 & $<0.05$ \\
IPF & OCD & PF (SF-36) & -0.62 & $<0.05$ \\
\hline
\end{tabular}

Table 6. Correlation between age and quality of life.

\begin{tabular}{lcc}
\hline Age $v$ s. Quality of life & $\mathrm{r}$ & $\mathrm{P}$ \\
\hline Impact (SGRQ) & 0.26 & $<0.05$ \\
Vit (SF-36) & -0.38 & $<0.05$ \\
SF (SF-36) & -0.28 & $<0.05$ \\
MH (SF-36) & -0.24 & $<0.05$ \\
\hline
\end{tabular}

SF-36 domains: vitality, mental health, impact of life, and social functioning (Table 6).

\section{DisCUSSION}

In the last decades, there has been an increasing interest in measuring the quality of life in both psychology and medicine, including pulmonary diseases. Quality of life questionnaires are called generic when they cover such aspects as emotional and social functioning, daily activities, and working capability. Questionnaires can also be disease specific. Those pertaining to the pulmonary system refer to such symptoms as cough, expectorating sputum, physical endurance, or dyspnea. Although disease specific tests are more sensitive in assessing symptoms, generic tests have some useful advantages. They allow comparing the quality of life in different diseases and populations. The SF-36 questionnaire is one such survey accepted for use by a number of health organizations [3].

Most significant quality of life impairment in patients awaiting lung transplantation was found in similar domains in both questionnaires: activity evaluated by SGRQ and RP (SF-36). Similar results have been obtained by Rodrique et al [4] whose study shows that in patients awaiting lung transplantation physical activity is the most decreased. Quality of life evaluation in relation to the diagnosis and dyspnea evaluation depend on the questionnaire used. In the present study, global SGRQ score indicated the biggest quality of life impairment in IIP patients. In SF-36, the cumulative score is divided into physical and mental health and it decreased the most in COPD patients.
All tests which evaluate dyspnea in the present study indicated its increase in patients qualified to lung transplantation. Various levels of dyspnea in patients with different diagnoses depended on the type of test used. Nevertheless, three out of four tests (MRC, OCD, and Borg scale) showed the strongest dyspnea in IIP patients. The study revealed a significant negative correlation between an increase in dyspnea perception and quality of life in the study group. This correlation was the strongest among domains referring to similar aspects of life. Dyspnea evaluated by the MRC scale affected PF, the OCD scale, which determines oxygen uptake for various activities, showed physical activity and PF being affected. The Borg scale and Baseline Dyspnea Index, which describe dyspnea in more detailed way, indicated the strongest correlation with Impacts in SGRQ. These results were similar in all patients independent of the diagnosis; correlations were only slightly changed. Interestingly, dyspnea did not increase with age. However, vitality, mental health, and social functioning decreased with age.

We conclude that the SF-36 and SGRQ questionnaires are sensitive tools in the assessment of the quality of life in patients referred for lung transplantation. Results achieved in this way can be helpful in determining disease severity, clinical symptoms, and functional impairment. The correlation found between the level of dyspnea and quality of life domains suggests that it would be worthwhile to add questions regarding dyspnea to assess the severity of the disease, clinical symptoms, and functional impairment during referring the patients for lung transplantation.

Conflicts of interest: No conflicts of interests were declared by the authors in relation to this article.

\section{REFERENCES}

1. Orens JB, Estenne M, Arcasoy S, Conte JV, Corris, Egan JJ, Egan T, Keshavjee S, Knopp C, Kotloff R, Martinez FJ, Nathan S, Palmer S, Patterson A, Singer L, Snell G, Studer S, Vachiery JL, Glanville AR. International Guidelines for the selection of lung transplant candidates: 2006 Update-A consensus report from the Pulmonary Scientific Council of the International Society for Heart and Lung Transplantation. J Heart Lung Transplant 2006; 7: 745-55.

2. Jastrzebski D, Kozielski J, Banas T, Cebula A, Gumola A, Ziora D, Krzywiecki A. Quality of life during one-year observation of patients with idiopathic pulmonary fibrosis awaiting lung transplantation. J Physiol Pharmacol 2005; 56 Suppl 4: 99-105.

3. Kaplan RM, Ries AL, Relly J, Mohsenfar Z. Measurement of health-related quality of life in the national emphysema trial. Chest 2004; 126: 781-9.

4. Rodrique JR, Baz MA. Waiting for lung transplantation: quality of life, mood, caregiving strain and benefit, and social intimacy of spouses. Clin Transplant 2007; 21: 722-7.

Address for correspondence:

D. Jastrzebski

Department Lung Diseases and Tuberculosis

Silesian Medical University

1, Koziolka St., 41-803 Zabrze

Poland

Phone/fax: +48 322745664

E-mail: darekjdr@poczta.onet.pl 